Journal of

Molecular Microbiology

and Biotechnology
DOI: $\underline{10.1159 / 000331009}$

\title{
Antimicrobial Peptides - Promising Alternatives to Conventional Antibiotics
}

\author{
Sylvia A. Baltzer Melissa H. Brown \\ School of Biological Sciences, Flinders University, Adelaide, S.A., Australia
}

\section{Key Words}

Antimicrobial resistance $\cdot$ Membrane modification • Peptide structure $\cdot$ Therapeutic agents $\cdot$ Antibiotics

\begin{abstract}
Antimicrobial peptides (APs) have been described as evolutionary ancient weapons. Produced by a wide variety of organisms as part of a non-specific immune response, these peptides are involved in the direct destruction of various microorganisms. Several APs have been shown to have broad activity spectra against microorganisms such as Gram-positive and Gram-negative bacteria, enveloped viruses, fungi and parasites. Given that resistance to a number of antibiotics has developed in a wide range of microbes, the potential of APs as novel therapeutic agents is being evaluated. However, optimisation of APs designed for therapy will need to focus on such factors as their susceptibility to proteolytic degradation and reduction of toxicity to mammalian cells. Strict guidelines pertaining to their use should also be established to prevent or hinder future development of bacterial resistance to such peptides.

Copyright $\odot 2011$ S. Karger AG, Basel
\end{abstract}

\section{Introduction}

It is a well-established fact that bacterial resistance to various antimicrobial agents has developed as a direct response to their exposure [Kaufmann and Hung, 2010].
Mechanisms involved in such resistance include: alteration of the target protein by mutation or enzymatic activation, the acquisition of genes from other bacterial species encoding for less susceptible target proteins, bypassing of the target protein, or the extrusion of the antimicrobial from the cell. These adaptations can arise in susceptible bacteria as a result of mutations or through horizontal gene transfer, either within or between genera, primarily employing mobile genetic elements such as plasmids, transposons or integrons.

The fact that antibiotic resistance continues to develop in a wide range of microbes has been well documented [Hawkey, 2008; Hawkey and Jones, 2009; Livermore, 2003]. Contributing factors to this development include the inappropriate use of antibiotics, such as the overuse of powerful, broad-spectrum antibiotics, the presence of antibiotics in the food/livestock industry [Arnold, 2007; Levy, 1998; Monroe and Polk, 2000], and from the inclusion of antimicrobials in household products [Aiello and Larson, 2003]. As a result of these strategies, many strains of pathogenic bacteria are now multidrug-resistant (defined as resistant to three or more antibacterial drug classes) and consequently problematic to treat. This represents a real threat to human health and has led to efforts to develop new antimicrobial agents, including alternative drugs based on antimicrobial peptides [Hancock, 2001; Lata et al., 2007].

Antimicrobial peptides (APs) have been described as evolutionary ancient weapons [Zasloff, 2002]. Found both in the animal and plant kingdoms, they play a fundamen-

\section{KARGER \\ Fax +4161306 1234 E-Mail karger@karger.ch} www.karger.com

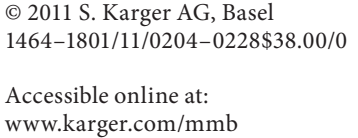


Fig. 1. A wheel plot showing the distribution of amino acids of a hypothetical model peptide. This model peptide takes on an $\alpha$-helical structure, with a distribution of positively charged amino acids on one side of the molecule and hydrophobic amino acids on the other side.

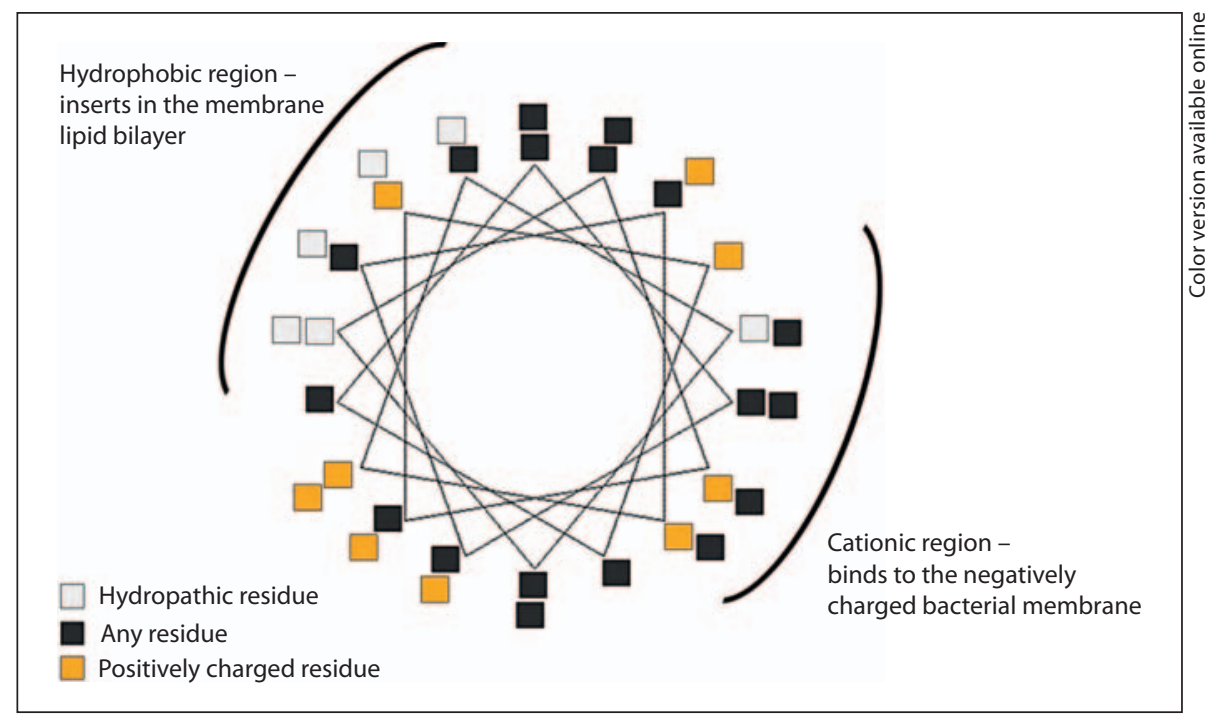

tal role in the innate non-specific defence system which confers resistance against infections without prior exposure to foreign pathogens [Brown et al., 2007; Hancock, 2001]. These responses are genetically predetermined and are possessed by all multicellular organisms [Izadpanah and Gallo, 2005]. Although diverse with respect to their amino acid sequence, their ability to form amphipathic conformations allows APs to interact and disrupt bacterial membranes [Powers and Hancock, 2003].

Considering their broad spectrum of activity against a range of bacteria, fungi, enveloped viruses and parasites [Brown and Hancock, 2006; Izadpanah and Gallo, 2005; Mohan et al., 2010; Vizioli and Salzet, 2002], APs have been the focus of studies looking at their potential as novel therapeutic agents. Their natural antimicrobial properties, selectivity, speed of action and a low propensity for the development of bacterial resistance make them ideal candidates for clinical development [Bradshaw, 2003]. Some concerns have however been shown regarding the emergence of resistance and cross-resistance, as even though resistance against APs is thought to be rare [Sahl and Peschel, 2006], bacterial pathogens have been shown to have developed countermeasures to limit the effectiveness of APs [Roy et al., 2009; Yount et al., 2006].

\section{Structure and Properties of Cationic Peptides}

APs are generally defined as having less than $50 \mathrm{ami-}$ no acid residues, a net positive charge and contain around 50\% hydrophobic amino acids [Hancock, 2001].
Their charge is largely due to an excess of positively charged basic amino acids such as lysine and arginine [Brown and Hancock, 2006]. APs are classed based on their secondary structures which include $\beta$-sheet peptides, stabilised by two to three disulphide bridges, amphipathic $\alpha$-helices, loop peptides formed from a single disulphide bridge, and extended structures. The most common of these are the $\beta$-sheet and $\alpha$-helical molecules [Hancock, 2001; Hancock and Diamond, 2000]. Although their secondary structures vary, they all share a common three-dimensional arrangement, as they fold into amphiphilic molecules that have charged and hydrophilic portions segregated from the hydrophobic portions [Hancock and Diamond, 2000]. This amphiphilic organisation, as shown in figure 1, allows for strong interactions between the positively charged peptides and negatively charged bacterial membranes, whilst the hydrophobic groups facilitate the penetration of the AP into the lipid phase of the membrane [Izadpanah and Gallo, 2005].

Although close to 900 AP sequences have been isolated from a wide range of organisms [http://bbcm.units. it/ tossi/amsdb.html], only a limited number have had their three-dimensional conformation determined. For example, $\beta$-sheet peptides such as tachyplesin, which are small 17-18 amino acid peptides isolated from the haemocytes of the Japanese horseshoe crab [Nakamura et al., 1988], are characterised by the presence of an antiparallel $\beta$-sheet predominately stabilised by disulphide bonds. Studies based on the structure-activity relationship of such $\beta$-sheet peptides have shown that these stabilising 


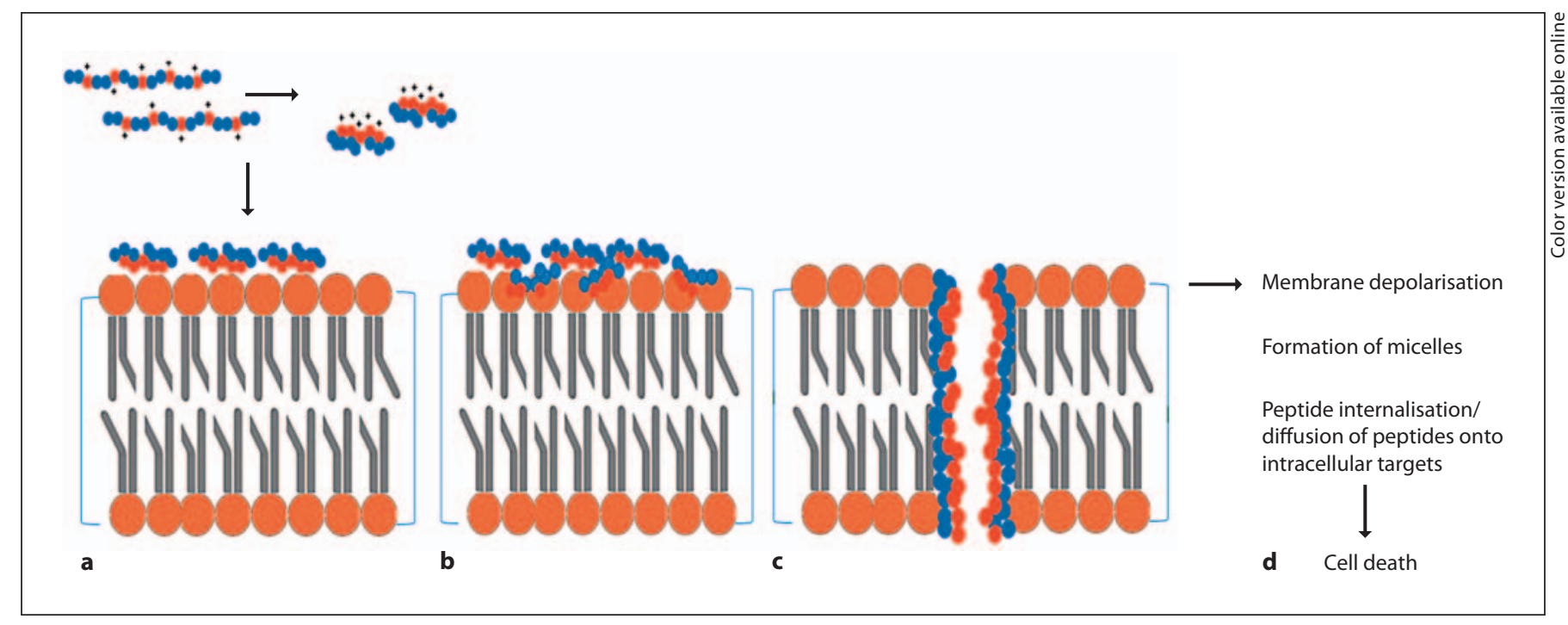

Fig. 2. The SMH model for the mechanism of action. Diagrammatic representation of peptide aggregation and interaction with the cell membrane (a), peptide-induced lipid displacement (b) and insertion of peptides into the membrane lipid bilayer (c) followed by eventual cell death (d). See text for more details.

disulphide bonds, although not necessarily required for antimicrobial activity, are essential for membrane translocation [Powers and Hancock, 2003].

\section{Mechanism of Action}

The exact nature of the mechanism of action of APs is still unclear [Appelt et al., 2007]. There are, however, studies which have indicated that their mode of action is specifically based on their structural properties. It is thus their sequence, size, cationic nature, hydrophobicity and amphipathicity that govern their interaction with target cells [Keymanesh et al., 2009]. In fact, it would appear that it is the fundamental difference in the design of membranes of multicellular organisms and microbes which APs have targeted [Zasloff, 2002]. Simply said, it is the organisation of bacterial membranes and their heavily populated lipid bilayer with their negatively charged phospholipid headgroups, which make bacteria vulnerable to APs. Eukaryotic membranes, on the other hand, contain predominantly neutral phospholipids and as a result are less susceptible to disruption by cationic peptides [Gottler and Ramamoorthy, 2009].

A model which is used to explain the antimicrobial action, or more specifically the initial interaction between APs and bacterial membranes, is the Shai-Matsuzaki-
Huang (SMH) model [Huang, 2000; Matsuzaki, 1999; Shai, 1999; Yang et al., 2000] (fig. 2). It has been proposed that unstructured peptides adopt a three-dimensional structure upon the interaction with the bacterial membrane and fold into amphiphilic molecules, with positively charged sides directly interacting with the anionic lipid headgroups of the bacterial membrane (fig. 2a). The lipids are then displaced by the peptides, causing a thinning of the outer leaflet of the bacterial membrane (fig. 2b). Following this, peptides aggregate and form a channel which allows for peptide translocation into the interior of the target cell (fig. 2c) [Straus and Hancock, 2006]. It should be noted, however, that the exact process of channel formation and peptide uptake is still unknown and at present a matter of controversy [Alves et al., 2009]. Moreover, there are three different models which have been proposed to explain the method of pore formation; these include the barrel-stove model, the torodial pore (wormhole) model and the micellisation model [Gottler and Ramamoorthy, 2009; Hancock et al., 2006]. There does not appear to be one model that fits all peptides, with various factors contributing to the process of pore formation.

The actual antimicrobial activity in order to kill bacteria resides in part in the disruption of the bacterial cell membrane which can occur in a number of ways. This includes membrane depolarisation, creation of pores (mi- 
celles) which could cause cellular contents to leak out, degradation of cell walls, and alteration of the lipid composition in the membrane bilayer which could lead to the disturbance of membrane functions [Straus and Hancock, 2006; Zasloff, 2002]. However, APs can also act intracellularly and activate lethal processes which can induce hydrolysis that can degrade the bacterial cell wall; furthermore, they may also damage critical intracellular targets resulting in cell death [Zasloff, 2002].

\section{Bacterial Resistance: A Probability?}

It has been speculated that unlike currently used antibiotics such as penicillin, acquisition of resistance by $\mathrm{mi}$ crobes against APs is thought to be improbable [Zasloff, 2002]. However, despite this somewhat optimistic perspective, an important factor to consider is whether or not resistance can be provoked [Marr et al., 2006]. Resistance is thought to be unlikely due to the mandatory interaction between peptides and the bacterial membrane. Thus, resistance would require the reconfiguration of the bacterial membrane, an energetically costly manoeuvre [Zasloff, 2002]. There is also the matter of peptides having numerous targets, making the elimination of one target less significant [Marr et al., 2006]. Regardless, bacterial resistance against certain peptides does exist, as shown in the examples below.

Although thought to be improbable, alteration of bacterial membranes has been shown as a mechanism of resistance. Such alterations include the incorporation of components with reduced anionic charge which leads to the inability of peptides to aggregate on bacterial membranes, preventing them from entering the cell. For instance, studies have shown that Staphylococcus aureus modifies the anionic phospholipids in the cytoplasmic membrane with L-lysine, resulting in a reduction of the net negative charge of the bacterial membrane and leading to the repulsion and subsequent resistance to APs [Peschel and Collins, 2001]. Similarly, modification of the lipopolysaccharide (LPS) of Gram-negative bacteria is yet another bacterial mechanism contributing to resistance [Gunn, 2001; Guo et al., 1998]. Modification of the lipid A component of LPS has been seen to result in the development of resistance against APs in such pathogens as Salmonella enterica serovar Typhimurium [Gunn, 2001; Peschel, 2002]. These modifications include the incorporation of fatty acids, thereby reducing the permeability of the outer membrane and increasing membrane structural stability [Peschel, 2002]. Furthermore, addition of phosphoethanolamine and aminoarabinose to the LPS core and lipid A regions, as well as the acetylation of the $\mathrm{O}$-antigen, contribute to resistance to APs [Gunn, 2001]. All of the above are induced by the PhoP-PhoQ two-component signal transduction pathway. This pathway controls the activation of a number of genes, including the pmrCAB locus encoding another two-component regulatory system also involved in the above-mentioned LPS modifications [Gunn et al., 1998]. Studies have shown that the PhoP-PhoQ regulatory system is required for resistance against a number of structurally different APs and that PhoP-PhoQ homologues are present in many, if not all, Gram-negative bacteria [Bell and Gouyon, 2003; Shi et al., 2004].

As can be seen, a common theme in AP resistance is the reduction of the net negative charge of the bacterial membrane. However, in addition to the above-mentioned modifications, bacteria can also alter their cell wall hydrophobicity and membrane fluidity, which also plays a role in the protection against APs [Bayer et al., 2000; Peschel, 2002]. Other contributors to resistance include membrane-bound efflux pumps, such as the $m \operatorname{tr} C D E$ efflux pump possessed by Neisseria gonorrhoeae, which exports APs from the cell [Shafer et al., 1998]. Lastly, peptides that do manage to enter the cell can be targeted by outer-membrane proteases and cleaved, as has been shown to occur in S. enterica serovar Typhimurium [Guina et al., 2000].

The type of resistance discussed above is deemed to be a component of pathogenesis of different bacteria and varies from the type of resistance that manifests itself as a result of prolonged exposure to therapy, as is the case with antibiotics. With reference to APs, little is known regarding acquired resistance as APs are yet to be used as therapeutic agents over a prolonged period of time [Perron et al., 2006].

\section{Acquired Resistance}

The evolution of resistance depends primarily on the relationship between the frequency of exposure of the treatment and the biological cost of resistance. In the case of APs, the cost of resistance is thought to pertain to the ability of bacteria to modify the bacterial cell membrane, which is metabolically expensive [Zasloff, 2002]. However, acquired resistance resulting from repeated exposure to APs has been demonstrated [Perron et al., 2006]. Through continued selection in the laboratory, 30 out of 32 lineages of Escherichia coli and Pseudomonas fluores- 
cens independently evolved heritable mechanisms of resistance. As a consequence, like conventional antibiotics, therapeutic use of APs may lead to the development of resistance amongst bacteria. Furthermore, it has been theorised that the wide variety of APs themselves may be an indication that they have evolved in response to bacterial co-evolution [Perron et al., 2006]. Thus, the reason why APs are so highly specific is that they must match the range of microbes that they attack. They are effective only when rare and lose their effectiveness with the evolution of resistance, which may be as a result of frequency of exposure.

\section{Cross-Resistance}

The development of bacterial cross-resistance against APs may also cast a cloud over APs promising role as alternative antimicrobial agents. Such resistance results when bacteria develop/obtain a resistance mechanism that is effective not just against a single agent, but against a number of antimicrobial molecules, in this case, a number of possibly structurally related APs from various organisms.

There are two different points of view regarding the possible use of APs as therapeutic agents, those who believe that they represent a promising alternative to current antibiotics and those who believe we may be playing with fire if we go ahead with their usage. The concern appears to stem from the fact that APs, as mentioned, are a part of the host defence system of animals and plants, implying that they already play a vital role in fighting off microbial invaders amongst other things, and as such are indispensible. Thus, should cross-resistance occur, we may inadvertently be sabotaging our own defence system [Bell and Gouyon, 2003]. Nevertheless, given that there is no known universal mechanism of resistance to APs, should resistance develop to a given therapeutic peptide, it will not necessarily lead to resistance to all host defence peptides [Hancock, 2003]. Regardless, given the possibility of this threat, it has been proposed that peptides designated for therapeutic use should be explored on a caseby-case basis. Finally, it should be noted that APs are not the only mechanism utilised by the host defence system. Thus, the proposal that resistance to APs may compromise the natural host defence system is thought to be extreme.

At this point it is too early to say what is possible and what is not. However, studies looking at induced resistance to certain APs have shown the development of low- level cross-resistance to human APs [Moskowitz et al., 2004] as well as other non-human APs [Samuelsen et al., 2005]. What is clear is that more research needs to be undertaken to examine both resistance, as well as cross-resistance to APs.

\section{Possible Therapeutic Application}

There are a number of factors that make APs ideal candidates for the generation of new antimicrobials. They are relatively small and thus easy to synthesize, fast acting, have a broad spectrum of activity against bacteria and a low propensity for the development of resistance [Bradshaw, 2003; Cherkasov et al., 2009; Hancock and Sahl, 2006; Marr et al., 2006]. They could potentially be used as single antimicrobials, in combination therapy, as immunomodulators or as endotoxin-neutralising agents [Marr et al., 2006]. Studies have shown than in vitro APs have excellent antimicrobial activity and represent a promising novel class of antibiotics. There also appears to be evidence that good in vitro activity may be indicative of efficient in vivo activity in animals [Hancock, 2001]. There are, however, hurdles which must first be overcome before their therapeutic usage can be implemented. These include difficulties with delivery, stability and susceptibility to proteases, unwanted toxicity against eukaryotic cells, the high cost of large-scale production and possible development of allergies [Bradshaw, 2003; Cherkasov et al., 2009; Hancock and Sahl, 2006]. It is also important to solve the secondary structure of each AP in order to understand its function and mode of action, only then can potent peptides be developed for pharmaceutical applications [Gottler and Ramamoorthy, 2009].

Although not many, there are examples of APs created, and in some cases approved, for clinical use. A 'firstgeneration' AP that is a variant of the amphibian peptide magainin, known as pexiganan, is a synthetic peptide of 22 amino acids and was manufactured to aid wound healing [Moore, 2003]. Although shown to be relatively nontoxic and effective, it was nevertheless rejected by the Food and Drug Administration (FDA) as it was considered to be no more effective than current available treatment [Moore, 2003]. There are, however, APs which have FDA approval and are currently in use as therapeutic agents; these are gramicidin S and polymyxin B. Both are ingredients in topical creams and solutions, but unfortunately their use is limited to topical applications only as both tend to be toxic [Hancock, 2001]. APs have also been 
shown to be effective in other non-medical applications. Nisin, a polypeptide produced by certain strains of $\mathrm{Lac}$ tococcus lactis, was approved as a food preservative in 1969 [Delves-Broughton et al., 1996]. Since then, it has been used to inhibit the activity of a wide range of Grampositive bacteria in a broad range of food products [Delves-Broughton, 2005]. Although in use, nisin is not ideal as it is affected by such factors as temperature and by additives such as sodium chloride; however, these factors also affect other preservatives [Keymanesh et al., 2009].

Combination therapy, the use of APs in combination with antibiotics, is another avenue being explored. Promising in vitro results have been found using D-9-mer (diastereomer) peptides against methicillin-resistant $S$. aureus and $P$. aeruginosa [Iwasaki et al., 2007]. These synthetic peptides, derived from insect defensins, have been shown to exhibit synergistic or additive effects when combined with antibiotics. By interacting with the bacterial membrane, they are thought to increase membrane permeability and allow antibiotics easier access to cytoplasmic targets [Iwasaki et al., 2007]. Another exciting characteristic of some APs is their ability to inhibit antibiotic efflux pumps preventing the expulsion of antibiotics [Renau et al., 1999; Tanabe et al., 2007]. Although APs may have a bright future as therapeutic agents, significant work is still required to explore possible harmful in vivo effects and to determine combinations of APs and antibiotics.

\section{Learning from the Past}

The mantra regarding the resulting problems caused by antibiotic overuse has been reported in both the lay and medical press over the past 15 years [Arnold, 2007], yet it would appear that this is being drowned out, as antibiotic overuse/abuse appears to continue to this day. Factors, such as unnecessary antibiotic prescription, whether it be for viral infections or due to patient expectations, overuse of antibiotics in the food industry, addition of antimicrobials in household and hygiene products and general improper use, all contribute to antimicrobial resistance [Aiello and Larson, 2003]. The emergence of resistance also saw a decline in the rate of discovery of new antibiotics. The number of pharmaceutical companies working on antibiotics dropped and as a result only a few completely new antibiotics reached the market. In addition, resistance is spreading from pathogenic bacteria found in hospital settings to those found circulating in the wider community, making antibiotic resistance a growing public concern [Fox, 2006].

It appears that as promising as APs may be for clinical use, we need to exercise some caution. Their possible applications seem endless, which can be a problem of its own. Not only can they be used clinically, but they can also be applied in agriculture, the food industry, animal husbandry and in aquaculture [Keymanesh et al., 2009]. Both a blessing and a curse, this widespread application may see us stumble into the same pitfall as we did with the use of antibiotics. Although potentially tempting, their use in such areas as the food industry should be regulated. Unnecessary additions to hygiene and household products should be avoided. Improving husbandry practice, setting up quarantines, vaccinations, inclusion of probiotics, proper diagnosis and control of viral infections could all lead to a reduced use of antibacterials [McEwen and Fedorka-Cray, 2002]. Inadequate antimicrobial treatment in the clinical environment must also be looked at. Using quicker methods of microbiological identification may limit the use of broad-spectrum antimicrobials and proper control of viral infections may prevent the onset of secondary bacterial infections which would also lead to a reduced need for antimicrobials [Kollef, 2000]. The possibilities to reduce antimicrobial resistance are certainly there, and whilst we may not be able to reverse the damage done with antibiotics [Spratt, 1996], we may just be lucky enough with APs and their possible applications in therapy.

\section{Conclusion}

The possibilities to develop APs into a new class of antibiotics are certainly promising. Their broad spectrum of activity, ease of synthesis and mechanism of action are all qualities which make them ideal candidates for therapeutic development and use. Nevertheless, there are still some limitations that need to be addressed before APs can be applied clinically. These include toxicity, stability and difficulties with delivery as well as high costs associated with large-scale production of therapeutic peptides. Only after these limitations are resolved can peptide-based therapeutics be fully realised. There are, however, companies which are working on these limitations and there is hope that with technological advancement, they will be resolved. We should be optimistic but also cautious. Studies of resistance in bacteria have shown that development of resistance is a continuous threat to any antimicrobial on the market. It is thus obvious that we need to plan a little 
bit better, learn from past mistakes and not simply replace one form of antibiotics with another without some form of regulation. The threat of bacteria developing cross-resistance against host defence peptides should also warrant caution, and thus we should consider potential bacterial resistance on a case-by-case basis prior to the indiscriminant use of APs as therapeutics.

\section{Acknowledgment}

S.A.B. is the recipient of an Australian Postgraduate Award. Work in our laboratory is supported in part by Project Grant 1002670 from the National Health and Medical Research Council (Australia).

\section{References}

-Aiello AE, Larson E: Antibacterial cleaning and hygiene products as an emerging risk factor for antibiotic resistance in the community. Lancet Infect Dis 2003;3:501-506.

-Alves ID, Correia I, Jiao CY, Sachon E, Sagan S, Lavielle S, Tollin G, Chassaing G: The interaction of cell-penetrating peptides with lipid model systems and subsequent lipid reorganization: thermodynamic and structural characterization. J Pept Sci 2009; 15:200-209.

-Appelt C, Schrey AK, Soderhall JA, Schmieder P: Design of antimicrobial compounds based on peptide structures. Bioorg Med Chem Lett 2007;17:2334-2337.

Arnold SR: Revenge of the killer microbe. Can Med Assoc J 2007;177:895-896.

-Bayer AS, Prasad R, Chandra J, Koul A, Smriti M, Varma A, Skurray RA, Firth N, Brown $\mathrm{MH}$, Koo SP, Yeaman MR: In vitro resistance of Staphylococcus aureus to thrombin-induced platelet microbicidal protein is associated with alterations in cytoplasmic membrane fluidity. Infect Immun 2000;68:35483553.

Bell G, Gouyon PH: Arming the enemy: the evolution of resistance to self-proteins. Microbiology 2003;149:1367-1375.

Bradshaw J: Cationic antimicrobial peptides: issues for potential clinical use. BioDrugs 2003; 17:233-240.

-Brown KL, Hancock RE: Cationic host defense (antimicrobial) peptides. Curr Opin Immunol 2006;18:24-30.

Brown KL, Mookherjee N, Hancock RE (eds): Antimicrobial, Host Defence Peptides and Proteins. Encyclopedia of Life Sciences. Chichester, Wiley, 2007, pp 1-11.

-Cherkasov A, Hilpert K, Jenssen H, Fjell CD, Waldbrook M, Mullaly SC, Volkmer R, Hancock REW: Use of artificial intelligence in the design of small peptide antibiotics effective against a broad spectrum of highly antibiotic-resistant superbugs. Chem Biol 2009; 4:65-74.

Delves-Broughton J: Nisin as a food preservative. Food Aust 2005;57:525-527.

Delves-Broughton J, Blackburn P, Evans RJ, Hugenholtz J: Applications of the bacteriocin, nisin. Antonie Van Leeuwenhoek 1996;69: 193-202.
Fox JL: The business of developing antibacterials. Nat Biotechnol 2006;24:1521-1528.

-Gottler LM, Ramamoorthy A: Structure, membrane orientation, mechanism, and function of pexiganan - a highly potent antimicrobial peptide designed from magainin. Biochim Biophys Acta 2009;1788:1680-1686.

Guina T, Yi EC, Wang HL, Hackett M, Miller SI: A PhoP-regulated outer membrane protease of Salmonella enterica serovar Typhimurium promotes resistance to $\alpha$-helical antimicrobial peptides. J Bacteriol 2000;182:40774086.

Gunn JS: Bacterial modification of LPS and resistance to antimicrobial peptides. J Endotoxin Res 2001;7:57-62.

-Gunn JS, Lim KB, Krueger J, Kim K, Guo L, Hackett M, Miller SI: PmrA-PmrB-regulated genes necessary for 4-aminoarabinose lipid A modification and polymyxin resistance. Mol Microbiol 1998;27:1171-1182.

Guo L, Lim KB, Poduje CM, Daniel M, Gunn JS, Hackett M, Miller SI: Lipid A acylation and bacterial resistance against vertebrate antimicrobial peptides. Cell 1998;95:189-198.

Hancock RE: Cationic peptides: effectors in innate immunity and novel antimicrobials. Lancet Infect Dis 2001;1:156-164.

Hancock RE: Concerns regarding resistance to self-proteins. Microbiology 2003;149:33433344.

-Hancock RE, Brown KL, Mookherjee N: Host defence peptides from invertebrates emerging antimicrobial strategies. Immunobiology 2006;211:315-322.

Hancock RE, Diamond G: The role of cationic antimicrobial peptides in innate host defences. Trends Microbiol 2000;8:402-410.

Hancock RE, Sahl HG: Antimicrobial and hostdefense peptides as new anti-infective therapeutic strategies. Nat Biotechnol 2006;24: 1551-1557.

Hawkey PM: The growing burden of antimicrobial resistance. J Antimicrob Chemother 2008;62(suppl 1):i1-i9.

-Hawkey PM, Jones AM: The changing epidemiology of resistance. J Antimicrob Chemother 2009;64(suppl 1):i3-i10.

Huang HW: Action of antimicrobial peptides: two-state model. Biochemistry 2000;39: 8347-8352.
Iwasaki T, Saido-Sakanaka H, Asaoka A, Taylor D, Ishibashi J, Yamakawa M: In vitro activity of diasterimeric antimicrobial peptides alone and in combination with antibiotics against methicillin-resistant Staphylococcus aureus and Pseudomonas aeruginosa. J Insect Biotechnol Sericol 2007;76:25-29.

Izadpanah A, Gallo RL: Antimicrobial peptides. J Am Acad Dermacol 2005;52:381-390.

Kaufmann BB, Hung DT: The fast track to multidrug resistance. Mol Cell 2010;37:297-298.

Keymanesh K, Soltani S, Sardari S: Application of antimicrobial peptides in agriculture and food industry. World J Microbiol Biotechnol 2009;25:933-944.

Kollef MH: Inadequate antimicrobial treatment: an important determinant of outcome for hospitalized patients. Clin Infect Dis 2000; 31:S131-S138.

Lata S, Sharma BK, Raghava GP: Analysis and prediction of antibacterial peptides. BMC Bioinf 2007;8:263.

Levy SB: Multidrug resistance - a sign of the times. N Engl J Med 1998;338:1376-1378.

Livermore DM: Bacterial resistance: origins, epidemiology, and impact. Clin Infect Dis 2003;36:S11-S23.

- Marr AK, Gooderham WJ, Hancock RE: Antibacterial peptides for therapeutic use: obstacles and realistic outlook. Curr Opin Pharmacol 2006;6:468-472.

Matsuzaki K: Why and how are peptide-lipid interactions utilized for self-defense? Magainins and tachyplesins as archetypes. Biochim Biophys Acta 1999;1462:1-10.

-McEwen SA, Fedorka-Cray PJ: Antimicrobial use and resistance in animals. Clin Infect Dis 2002;34:S93-S106.

Mohan KV, Rao SS, Atreya CD: Antiviral activity of selected antimicrobial peptides against vaccinia virus. Antiviral Res 2010;86:306-311.

-Monroe S, Polk R: Antimicrobial use and bacterial resistance. Curr Opin Microbiol 2000;3: 496-501.

Moore A: The big and small of drug discovery. Biotech versus pharma: advantages and drawbacks in drug development. EMBO Rep 2003;4:114-117. 
Moskowitz SM, Ernst RK, Miller SI: PmrAB, a two-component regulatory system of Pseudomonas aeruginosa that modulates resistance to cationic antimicrobial peptides and addition of aminoarabinose to lipid A. J Bacteriol 2004;186:575-579.

- Nakamura T, Furunaka H, Miyata T, Tokunaga F, Muta T, Iwanaga S, Niwa M, Takao T, Shimonishi Y: Tachyplesin, a class of antimicrobial peptides from the hemocytes of the horseshoe-crab (Tachypleus tridentatus) isolation and chemical structure. J Biol Chem 1988;263:16709-16713.

-Perron GG, Zasloff M, Bell G: Experimental evolution of resistance to an antimicrobial peptide. Proc R Soc B 2006;273:251-256.

Peschel A: How do bacteria resist human antimicrobial peptides? Trends Microbiol 2002;10: 179-186.

- Peschel A, Collins LV: Staphylococcal resistance to antimicrobial peptides of mammalian and bacterial origin. Peptides 2001;22:1651-1659.

- Powers JP, Hancock RE: The relationship between peptide structure and antibacterial activity. Peptides 2003;24:1681-1691.
Renau TE, Leger R, Flamme EM, Sangalang J, She MW, Yen R, Gannon CL, Griffith D, Chamberland S, Lomovskaya O, Hecker SJ, Lee VJ, Ohta T, Nakayama K: Inhibitors of efflux pumps in Pseudomonas aeruginosa potentiate the activity of the fluoroquinolone antibacterial levofloxacin. J Med Chem 1999;42:4928-4931.

Roy H, Dare K, Ibba M: Adaptation of the bacterial membrane to changing environments using aminoacylated phospholipids. Mol Microbiol 2009;71:547-550

-Sahl H, Peschel A: The co-evolution of host cationic antimicrobial peptides and microbial resistance. Nat Rev Microbiol 2006;4:529-536.

-Samuelsen O, Haukland HH, Jenssen H, Kramer M, Sandvik K, Ulvatne H, Vorland LH: Induced resistance to the antimicrobial peptide lactoferricin B in Staphylococcus aureus. FEBS Lett 2005;579:3421-3426.

Shafer WM, Qu X, Waring AJ, Lehrer RI: Modulation of Neisseria gonorrhoeae susceptibility to vertebrate antibacterial peptides due to a member of the resistance/nodulation/division efflux pump family. Proc Natl Acad Sci USA 1998;95:1829-1833.

Shai Y: Mechanism of the binding, insertion and destabilization of phospholipid bilayer membranes by $\alpha$-helical antimicrobial and cell non-selective membrane-lytic peptides. Biochim Biophys Acta 1999;1462:55-70.
Shi YX, Cromie MJ, Hsu FF, Turk J, Groisman EA: PhoP-regulated Salmonella resistance to the antimicrobial peptides magainin 2 and polymyxin B. Mol Microbiol 2004;53:229-241.

Spratt BG: Antibiotic resistance: counting the cost. Curr Biol 1996;6:1219-1221.

Straus SK, Hancock RE: Mode of action of the new antibiotic for Gram-positive pathogens daptomycin: comparison with cationic antimicrobial peptides and lipopeptides. Biochim Biophys Acta 2006;1758:1215-1223.

- Tanabe K, Lamping E, Adachi K, Takano Y, Kawabata K, Shizuri Y, Niimi M, Uehara Y: Inhibition of fungal ABC transporters by unnarmicin $A$ and unnarmicin $C$, novel cyclic peptides from marine bacterium. Biochem Biophys Res Commun 2007;364:990-995.

Vizioli J, Salzet M: Antimicrobial peptides versus parasitic infections? Trends Parasitol 2002;18:475-476.

Yang L, Weiss TM, Lehrer RI, Huang HW: Crystallization of antimicrobial pores in membranes: magainin and protegrin. Biophys J 2000;79:2002-2009.

-Yount NY, Bayer AS, Xiong YQ, Yeaman MR: Advances in antimicrobial peptide immunobiology. Biopolymers 2006;84:435-458.

Zasloff M: Antimicrobial peptides of multicellular organisms. Nature 2002;415:389-395. 